\title{
Inversion of the domain wall propagation in synthetic ferrimagnets
}

A. Hamadeh, P. Pirro, J.-P. Adam, Y. Lu, M. Hehn, S. Petit Watelot, and S. Mangin

Citation: Appl. Phys. Lett. 111, 022407 (2017);

View online: https://doi.org/10.1063/1.4993604

View Table of Contents: http://aip.scitation.org/toc/apl/111/2

Published by the American Institute of Physics

\section{Articles you may be interested in}

Investigation of the Dzyaloshinskii-Moriya interaction and room temperature skyrmions in $\mathrm{W} / \mathrm{CoFeB} / \mathrm{MgO}$ thin films and microwires

Applied Physics Letters 111, 022409 (2017); 10.1063/1.4991360

Chopping skyrmions from magnetic chiral domains with uniaxial stress in magnetic nanowire

Applied Physics Letters 111, 022406 (2017); 10.1063/1.4993433

Indirect excitation of self-oscillation in perpendicular ferromagnet by spin Hall effect

Applied Physics Letters 111, 022410 (2017); 10.1063/1.4991663

Making the Dzyaloshinskii-Moriya interaction visible

Applied Physics Letters 110, 242402 (2017); 10.1063/1.4985649

Fast switching and signature of efficient domain wall motion driven by spin-orbit torques in a perpendicular anisotropy magnetic insulator/Pt bilayer

Applied Physics Letters 111, 072406 (2017); 10.1063/1.4994050

Field-free spin-orbit torque switching of composite perpendicular $\mathrm{CoFeB} / \mathrm{Gd} / \mathrm{CoFeB}$ layers utilized for threeterminal magnetic tunnel junctions

Applied Physics Letters 111, 012402 (2017); 10.1063/1.4990994

\section{Scilight}

Sharp, quick summaries illuminating the latest physics research

\section{Sign up for FREE!}

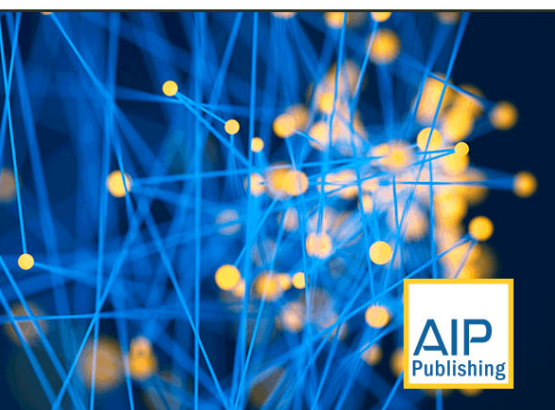




\title{
Inversion of the domain wall propagation in synthetic ferrimagnets
}

\author{
A. Hamadeh, ${ }^{1}$ P. Pirro, ${ }^{1, a)}$ J.-P. Adam, ${ }^{2}$ Y. Lu, ${ }^{1}$ M. Hehn, ${ }^{1}$ S. Petit Watelot,${ }^{1}$ and S. Mangin ${ }^{1}$ \\ ${ }^{1}$ Institut Jean Lamour, Université Lorraine, UMR 7198 CNRS, 54506 Vandoeuvre-lès-Nancy, France \\ ${ }^{2}$ Centre de Nanosciences et de Nanotechnologies, CNRS, Univ. Paris-Sud, Université Paris-Saclay, \\ C2N - Orsay, 91405 Orsay cedex, France
}

(Received 20 April 2017; accepted 24 June 2017; published online 12 July 2017)

\begin{abstract}
We report on magnetic domain wall velocity measurements in a synthetic ferrimagnet made of two perpendicular ferromagnetic layers antiferromagnetically exchange coupled. In this system, two types of transitions may be observed: one from a parallel alignment to an antiparallel alignment of the magnetization of the two layers and the other between the two possible antiparallel alignments. Those transitions are shown to be dominated by domain wall propagation. The domain wall velocity as a function of the applied magnetic field pulse amplitude has been measured. Two remarkable features are observed: first, a drastic breakdown of the domain wall velocity and then an inversion of the domain propagation direction are observed when the field pulses reach values comparable to the exchange field between the two layers. This unexpected behavior can be understood qualitatively using a simple model taking into account the competition between interlayer exchange coupling and the external driving field. Published by AIP Publishing.

[http://dx.doi.org/10.1063/1.4993604]
\end{abstract}

Domain wall motion in thin magnetic films offers interesting opportunities to build high performance spintronic devices. ${ }^{1}$ Widely recognized examples are racetrack memories, ${ }^{2,3}$ domain wall logic circuits, ${ }^{4}$ and domain wall nano-oscillators. ${ }^{5}$ Quite recently, it has been proposed that synthetic antiferromagnets and ferrimagnets with perpendicular-to-plane magnetized layers can provide an interesting solution for the operation of racetrack memories since domain walls in these systems can reach high velocities. ${ }^{9}$ In addition, they show an almost vanishing magnetic stray field, which is useful for integrated circuits because the stray field is the primary obstacle for closely packed domain walls. ${ }^{6,7}$ With future spintronic applications in mind, micromagnetic simulation or experimental studies in synthetic antiferromagnets have been focussed on domain wall movement through DC currents. ${ }^{7-9}$ However, the understanding of the domain wall dynamics in these systems under the impact of magnetic fields is crucial to predict the stability and robustness of future devices. This is especially true if the antiferromagnetically exchange coupled layer system is not fully compensated (synthetic ferrimagnet) or if the coupling strength between the layers is not strong enough to exclude a deviation from the antiferromagnetic coupling during the motion. Last but not least, the magnetic field driven motion also helps to understand the domain wall dynamics from a more fundamental point of view. ${ }^{10-13}$

In this letter, we present an experimental study of domain wall motion in a multilayer system consisting of two antiferromagnetically exchange coupled $\mathrm{CoFeB}$ layers of different thicknesses. In this synthetic ferrimagnetic system, the magnetic domain walls move under the action of an applied driving field pulse $\mathrm{B}_{\text {pulse. }}$. The important result of this work is that the velocity-field characteristic of the domain wall

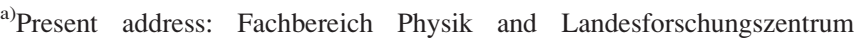
OPTIMAS, Technische Universität Kaiserslautern, 67663 Kaiserslautern, Germany.
}

motion shows three regimes: (1) the low field regime where the velocity increases as a function of the field pulse intensity, (2) the intermediate regime where the velocity decreases as the pulse intensity is increased, and (3) the large field regime where the direction of the domain displacement is inverted compared to the two previous regimes.

The heterostructure, shown in the inset of Fig. 1, consists of two ultra-thin ferromagnetic $\mathrm{CoFeB}$ layers with strong perpendicular anisotropy which are antiferromagnetically exchange coupled. The multilayer stack, $\mathrm{MgO}(0.5 \mathrm{~nm}) /$ $\mathrm{CoFeB}(1.1 \mathrm{~nm}) / \mathrm{Ta}(0.75) / \mathrm{CoFeB}(0.8 \mathrm{~nm}) / \mathrm{MgO}(1.0 \mathrm{~nm})$, was directly grown on a GaAs substrate. The magnetic properties of our system have been determined from an extensive study performed using a superconducting quantum interference device (SQUID), inductive ferromagnetic resonance frequency (FMR), and Brillouin light scattering spectroscopy

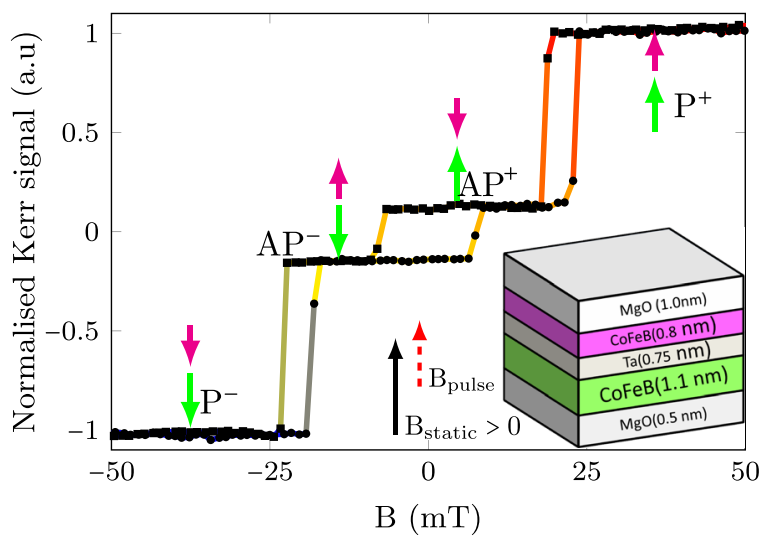

FIG. 1. Normalised Kerr signal as a function of the applied magnetic field along the out of the plane direction. Four possible magnetic states can be observed corresponding to four magnetic configurations: two $\left(\mathrm{P}^{+}\right.$and $\left.\mathrm{P}^{-}\right)$ where the two magnetizations are parallel to each other and two $\left(\mathrm{AP}^{+}\right.$and $\mathrm{AP}^{-}$) where the two magnetizations are antiparallel to each other. The inset shows a sketch of the studied synthetic ferrimagnet made of two CoFeB layers which are separated by a $\mathrm{Ta}(0.75 \mathrm{~nm})$ layer and interacting via antiferromagnetic interlayer exchange coupling. 
measurement on the same multilayer. ${ }^{16}$ The magnetocrystalline anisotropy of the layers and the exchange coupling could be determined. ${ }^{16}$ Figure 1 shows the evolution of the magnetization of the stack along the field axis measured by polar magneto-optical Kerr microscopy (MOKE) as a function of the applied out-of-plane field. The obtained hysteresis loop is comparable to the one observed for other antiferromagnetically exchange coupled bilayers. ${ }^{17,18}$ The arrows indicate the relative orientations of the magnetization in each layer: magenta (light green) arrows for the thin (thick) layer magnetization. It can be seen from the major loop that the two $\mathrm{CoFeB}$ layers reveal a perpendicular magnetization. Starting from a positive saturation field of $50 \mathrm{mT}$, the step at $\sim 18 \mathrm{mT}$ can be identified as the switching of the $0.8 \mathrm{~nm}$ thin $\mathrm{CoFeB}$ layer to minimize the interlayer exchange energy. Thus, a switching from a parallel state $\mathrm{P}^{+}$to an anti-parallel state $\mathrm{AP}^{+}$takes place. Then, the next step at $\sim-5 \mathrm{mT}$ corresponds to the simultaneous switching of the two $\mathrm{CoFeB}$ layers to minimize the total Zeeman energy, and thus, a transition between the two AP states occurs (from $\mathrm{AP}^{+}$to $\mathrm{AP}^{-}$). Finally, the step at $\sim-23 \mathrm{mT}$ is again attributed to the thin $\mathrm{CoFeB}$ layer. Three types of magnetic transitions can be then be observed: $\mathrm{P}^{+} \rightarrow$ $\mathrm{AP}^{+}, \mathrm{P}^{+} \rightarrow \mathrm{AP}^{-}$, and $\mathrm{AP}^{-} \rightarrow \mathrm{P}^{-}$. Each of these transitions takes place by domain nucleation followed by domain wall propagation. From the switching field between the $\mathrm{AP} \rightarrow \mathrm{P}$ and $\mathrm{AP} \rightarrow \mathrm{AP}$ configurations, an interlayer exchange coupling field of $\mu_{0} H_{\mathrm{j}}=19.5 \mathrm{mT}$ has been extracted, which corresponds to an interlayer coupling constant of $11 \mu \mathrm{J} / \mathrm{m}^{2}$.

To investigate the domain wall dynamics in our system, we imaged the domains using a high resolution $(\sim 0.8 \mu \mathrm{m})$ MOKE microscope. The transition of $\mathrm{P}^{+}$to $\mathrm{AP}^{+}$was first studied. The system is saturated with a large positive field, and then, the static field is set to $B_{\text {static }}=+19.5 \mathrm{mT}$. At this point, the sample is in the $\mathrm{P}^{+}$configuration. Subsequently, reversed domains are nucleated in the thin $\mathrm{CoFeB}$ layer $(0.8 \mathrm{~nm})$ by the application of a negative low field pulse $(-2 \mathrm{mT})$ generated by an electromagnet. These nucleated regions in the $\mathrm{AP}^{+}$are stable for $\mathrm{B}_{\text {static }}=+19.5 \mathrm{mT}$ [Fig. 1(b)]. The domains were then expanded using additional positive field pulses generated by a small coil mounted close to the sample surface, which can produce field pulses up to $+50 \mathrm{mT}$ with well-defined amplitude and variable duration. MOKE images after the nucleation and after the propagation of the domain (P/AP) are shown in Figs. 2(b) and 2(c). In order to determine the wall displacements, a static technique has been used in which images of the domain structure were taken at $\mathrm{B}_{\text {static }}$ before and after the application of the field pulse. These images were then subtracted one from another, and the displacement was measured. We repeated this measurement procedure for several field pulses of the same amplitude but different durations $(>5 \mu$ s to ensure a rectangle pulse shape). The velocity was then estimated from the slope of a linear fit of the dependence of the displacement data with the pulse durations. Further details regarding this method can be found in Ref. 15. Throughout this letter, positive (negative) velocities signify that the initially nucleated domain grows (collapses). The experimental velocity curves for the system at $B_{\text {static }}=+19.5 \mathrm{mT}$ are shown in Fig. 2(a). As it is shown in the inset of Fig. 2(a), the obtained velocity versus field pulse characteristics are

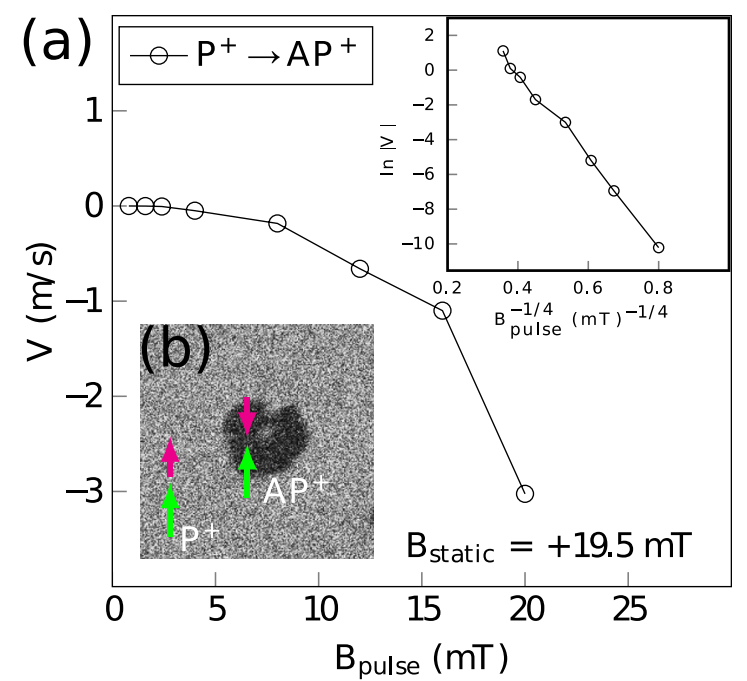

FIG. 2. Domain wall velocity (V) as a function of field pulse $B_{\text {pulse }}$ applied parallel to a static magnetic field $\left(\mathrm{B}_{\text {static }}=+19.5 \mathrm{mT}\right)$. Both fields are applied perpendicular to the film plane. The inset shows $\ln |V|$ versus $\mathrm{H}_{\text {pulse }}^{-1 / 4}$. (b) Kerr microscopy image of the magnetic configuration during a $\left(\mathrm{P}^{+} \rightarrow \mathrm{AP}^{+}\right)$transition. The arrows show the relative orientations of the magnetization in the thinner (resp. thicker) layer in magenta (resp. green). The domain has been first nucleated under a static field of $\left(\mathrm{B}_{\text {static }}=+19.5 \mathrm{mT}\right)$, and then, the domain was expanded using a field pulse.

qualitatively consistent with a creep motion of the domain walls, which predicts a linear dependence of $\ln |V|$ on $\mathrm{B}_{\text {pulse }}^{-1 / 4}{ }^{14}$ Here, the thick layer is kept positively saturated along the direction of $B_{\text {static. }} B_{\text {pulse }}$ shrinks the size of the inverted domain in the thin layer (negative domain wall velocity). This can be explained by the tendency of the system to reduce its Zeeman energy during the application of the field pulse. For field pulses larger than $20 \mathrm{mT}$, the nucleation of additional domains takes place, and it is impossible to measure the domain wall velocities.

We now focus on the $\mathrm{AP}^{+}$to $\mathrm{AP}^{-}$and $\mathrm{AP}^{+}$to $\mathrm{AP}^{-}$transitions, where two coupled domain walls are present, one in each layer. To do so, we first saturate both layers in the positive (resp. negative) field direction, and then, a short negative (resp. positive) nucleating field pulse was applied with an amplitude and duration such that a $\mathrm{AP}^{+}\left(\right.$resp. $\left.\mathrm{AP}^{-}\right)$domain is nucleated in an $\mathrm{AP}^{+}$surrounding (resp. $\mathrm{AP}^{-}$). Those reversed domains are stable in the zero static field $\left(\mathrm{B}_{\text {static }}\right.$ $=0 \mathrm{mT}$ ). Similar to what was done in the first transition between $\mathrm{P}^{+}$and $\mathrm{AP}^{+}$, an image of the nucleated domains was then taken, and a positive field pulse was subsequently applied to displace the walls, after which a second image of the domain structure was taken. The wall velocity in the two layers is shown in Fig. 3(a), where we plot the results obtained for the two domain configurations $\mathrm{AP}^{+} \rightarrow \mathrm{AP}^{-}$and $\mathrm{AP}^{-} \rightarrow$ $\mathrm{AP}^{+}$. Three different regions can be distinguished in this figure. At field pulse amplitudes between 4 and $12 \mathrm{mT}$ (region (1), the absolute average wall velocity increases with increasing $\mathrm{B}_{\text {pulse }}$ and reaches a local maximum for $\mathrm{B}_{\text {pulse }}=12 \mathrm{mT}$. For higher amplitudes until $18-20 \mathrm{mT}$, the velocities decrease (region (2)) until the measured displacement reaches zero. As $\mathrm{B}_{\text {pulse }}$ is further increased towards higher field pulse amplitudes, the wall propagation direction is inverted.

To illustrate the processes occurring during the application of the magnetic field pulse, we sketched the domain 

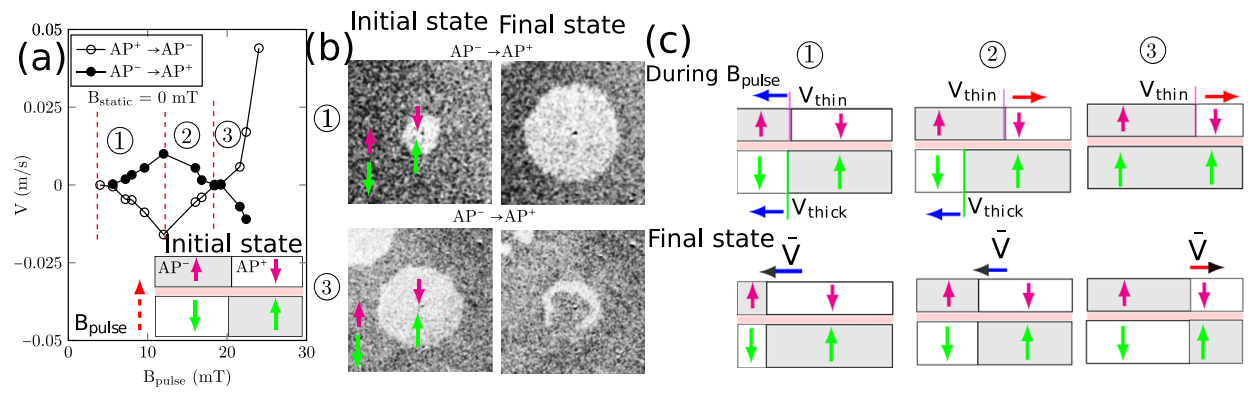

FIG. 3. (a) Domain-wall velocity versus field pulse amplitude $\left(\mathrm{B}_{\text {static }}=0 \mathrm{mT}\right)$. Filled symbols show velocities obtained for the case of an $\mathrm{AP}^{-}$to $\mathrm{AP}^{+}$transition $\left(\mathrm{AP}^{-} \rightarrow \mathrm{AP}^{+}\right.$) and open symbols those obtained for an $\mathrm{AP}^{+}$to $\mathrm{AP}^{-}$transition. (b) The MOKE images showing the magnetic contrast in the case of region (1) and region (3). In region (1), the domain grows, and in region (3), the domain shrinks. (c) Evolution of the magnetic configuration during the pulse and after the pulse when the system reaches its final state. The orientation in the thin and thick layers depends on the competition of the antiferromagnetic exchange coupling between the layers and the Zeeman energy. Horizontal arrows (red and blue) indicate the direction in which the domain walls move under the influence of positive applied field pulses.

walls in the upper and lower layers as shown in the crosssectional view of the $\mathrm{CoFeB}$ bilayer film in Fig. 3(c). In the initial state (before the field pulse), domain walls in the thin and thick layers are located at the center of the sketch. In the thin (thick) layer, there is a magnetization domain "up" ("down") on the left of the wall and a magnetization domain "down" ("up") on the right of the wall. The behavior of the three different field regions is shown schematically in Fig. 3 (c) where we have sketched the magnetic configuration during the pulse and after the pulse. In region (1), the Zeeman energy of the field pulse is small compared to the interlayer exchange coupling. The bilayer behaves as a rigidly coupled bilayer, and the domain walls inside the thin layer and thick layer show a coupled movement. The domain has a net magnetization along the field pulse direction growth to minimize the overall Zeeman energy. In region (2), the pulse amplitude becomes comparable to the exchange coupling, and the magnetization reversals in the two layers start to differ. The domain walls inside the thin layer and the thick layer move in opposite directions to minimize the Zeeman energy individually. After the pulse, the domain walls in the two layers move back to a common position to minimize the interlayer exchange. Thus, the net displacement depends on the difference of the two velocities $v_{\text {thin }}$ and $v_{\text {thick }}$ and the mobility of the walls after the field pulse, which leads to a weaker net displacement in comparison to the movement in region (1). In region (3), the pulse amplitude is so large that the magnetization of the thicker layers follows the external field, and the $\mathrm{AP}^{-}$configuration becomes a $\mathrm{P}^{+}$configuration. There is then only one domain wall in the thin layer which moves to minimize the Zeeman energy. Once the pulse is over, the $\mathrm{P}^{+}$ domain relaxes back to its original $\mathrm{P}^{-}$configuration. Thus, after the pulse, one can observe that the net displacement of the domain wall is in a direction opposite to the case of regions 1 and 2.

To achieve full field-velocity characteristics, we also performed measurements of the velocity versus $B_{\text {pulse }}$ at the transitions $\mathrm{P}^{-}$and $\mathrm{AP}^{-}$. Here, the dynamics of domain walls were measured under a negative static field with $B_{\text {static }}$ $=-19.5 \mathrm{mT}$, and positive field pulses were applied. Thus, in this case, the two fields point in opposite directions. The evolution of the domain wall velocity, which is shown in Fig. 4(a), is qualitatively very similar to the one observed at $\mathrm{B}_{\text {static }}=0 \mathrm{mT}$. In Fig. 4(a), the domain wall velocity regions observed in Fig. 3(a) are just shifted by the negative static field. This indicates that if the field pulse amplitude is larger than $\mathrm{B}_{\text {static }}=-19.5 \mathrm{mT}$, the $\mathrm{P}^{-}$domain is transformed to an $\mathrm{AP}^{-}$domain, whereas the initial $\mathrm{AP}^{-}$domain is transformed to an $\mathrm{AP}^{+}$domain. Thus, the domain wall movement simply depends on the sum $B_{\text {static }}+B_{\text {pulse }}$, and the same qualitative explanation can be given to explain the behavior of the measured net domain wall velocity.

To conclude, we have measured the domain wall velocity as a function of the field pulse amplitude for a synthetic ferrimagnet, showing strong perpendicular anisotropy, namely, $\mathrm{CoFeB} / \mathrm{Ta} / \mathrm{CoFeB}$. Four magnetic states are possible: two for which the two magnetizations are parallel $\left(\mathrm{P}^{+}\right.$ and $\mathrm{P}^{-}$) and two for which the two magnetizations are antiparallel $\left(\mathrm{AP}^{+}\right.$and $\left.\mathrm{AP}^{-}\right)$. We studied how domains grow or shrink depending on the combination of magnetic field pulses and the static magnetic bias field. We were able to distinguish different regimes depending on the field pulse intensity. For instance, when an antiparallel state (e.g., $\left.\mathrm{AP}^{+}\right)$is nucleated in the inverse antiparallel state $\left(\mathrm{AP}^{-}\right)$, then for a field pulse amplitude which is small in comparison to the exchange coupling field between the two layers, the domain grows, whereas for large field pulse amplitudes, the direction of the domain displacement is inverted. Thus, synthetic ferrimagnetic systems would be suitable for large domain velocity as predicted ${ }^{9}$ if the interlayer exchange coupling is strong

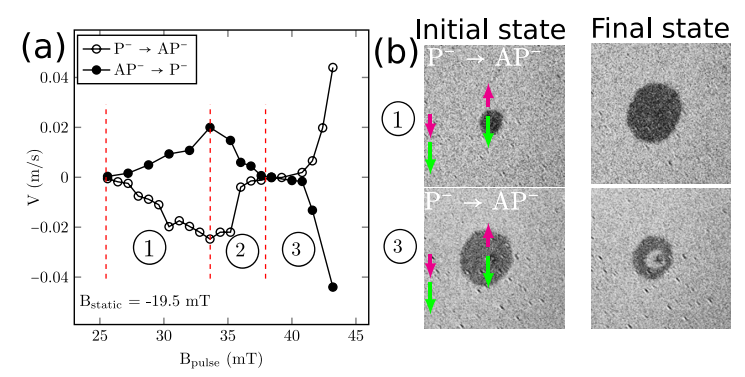

FIG. 4. Domain wall velocity under field pulses applied opposite to the static magnetic field $\left(\mathrm{B}_{\text {static }}=-19.5 \mathrm{mT}\right)$. The filled (open) circles correspond to a measurement when the nucleated domains are $\mathrm{AP}^{-}\left(\mathrm{P}^{-}\right)$. The orientation of the magnetization in the thin and thick layers is compared which is denoted by arrows in (b). Black regions correspond to $\mathrm{AP}^{-}$domains. Dark grey regions correspond to $\mathrm{P}^{-}$domains. (b) Examples of Kerr Images of the domain walls when the started nucleation DW grows or collapses. (b) Field pulse amplitudes and durations are (b-1)-) $28 \mathrm{mT}, 6 \mathrm{~ms}$ and (b-3)-) $32 \mathrm{mT}$, $1500 \mu \mathrm{s}$. 
enough to prevent a ferromagnetic alignment during the domain wall propagation.

This work was supported by the ANR-NSF Project, ANR13-IS04-0008-01, COMAG by the ANR-Labcom Project LSTNM and by the Université de la Grande Region (UniGR funded P. Pirro Post-Doc). Experiments were performed using equipment from the TUBE - Daum funded by FEDER (EU), ANR, the Region Lorraine, and Grand Nancy.

${ }^{1}$ S. A. Wolf, D. D. Awschalom, R. A. Buhrman, J. M. Daughton, S. von Molnarand, M. L. Roukes, A. Y. Chtchelkanova, and D. M. Treger, Science 294, 1488 (2001).

${ }^{2}$ S. Parkin, M. Hayashi, and L. Thomas, Science 320, 190 (2008).

${ }^{3}$ M. Hayashi, L. Thomas, R. Moriya, C. Rettner, and S. S. P. Parkin, Science 320, 209 (2008).

${ }^{4}$ D. A. Allwood, G. Xiong, C. C. Faulkner, D. Atkinson, D. Petit, and R. P. Cowburn, Science 309, 1688 (2005).

${ }^{5}$ J. He and S. Zhang, Appl. Phys. Lett. 90, 142508 (2007).

${ }^{6}$ P. Gawronski, V. Zhukova, A. Zhukov, and J. Gonzalez, J. Appl. Phys. 114, 043903 (2013).

${ }^{7}$ S.-H. Yang, K.-S. Ryu, and S. Parkin, Nature Nanotechnol. 10, 221 (2015).
${ }^{8}$ S. Lepadatu, H. Saarikoski, R. Beacham, M. J. Benitez, T. A. Moore, G. Burnell, S. Sugimoto, D. Yesudas, M. C. Wheeler, J. Miguel, and S. S. Dhesi, Sci. Rep. 7, 1640 (2017).

${ }^{9}$ M. Kuteifan, M. V. Lubarda, S. Fu, R. Chang, M. A. Escobar, S. Mangin, E. E. Fullerton, and V. Lomakin, AIP Adv. 6, 045103 (2016).

${ }^{10}$ J. Moritz, F. Garcia, J. C. Toussaint, B. Dieny, and J. P. Noziéres, Europhys. Lett. 65, 123 (2004).

${ }^{11}$ A. S. Balankin, R. G. Paredes, O. Susarrey, D. Morales, and F. C. Vacio, Phys. Rev. Lett. 96, 056101 (2006).

${ }^{12}$ P. J. Metaxas, R. L. Stamps, J.-P. Jamet, J. Ferré, V. Baltz, B. Rodmacq, and P. Politi, Phys. Rev. Lett. 104(23), 237206 (2010).

${ }^{13}$ A. Zhukov, J. M. Blanco, A. Chizhik, M. Ipatov, V. Rodionova, and V. Zhukova, J. Appl. Phys. 114, 043910 (2013).

${ }^{14}$ P. J. Metaxas, J. P. Jamet, J. Ferre, B. Rodmacq, B. Dieny, and R. Stamps, J. Magn. Magn. Mater. 320(21), 2571 (2008).

${ }^{15}$ N. Vernier, J.-P. Adam, S. Eimer, G. Agnus, T. Devolder, T. Hauet, B. Ocker, F. Garcia, and D. Ravelosona, Appl. Phys. Lett. 104(12), 122404 (2014).

${ }^{16}$ P. Pirro, A. Hamadeh, M. Lavanant-Jambert, T. Meyer, B. Tao, E. Rosario, Y. Lu, M. Hehn, S. Mangin, and S. Petit Watelot, J. Magn. Magn. Mater. 432, 260 (2017).

${ }^{17}$ S. M. Watson, T. Hauet, J. A. Borchers, S. Mangin, and E. E. Fullerton, Appl. Phys. Lett. 92, 202507 (2008).

${ }^{18}$ S. Mangin, T. Hauet, P. Fischer, D. H. Kim, J. B. Kortright, K. Chesnel, E. Arenholz, and E. E. Fullerton, Phys. Rev. B 78, 024424 (2008). 